IMTC 2005 - Instrumentation and Measurement

Technology Conference

Ottawa, Canada, May 17-19, 2005

\title{
Design and Manufacture of Surface Acoustic Wave Sensors for Real-Time Weigh-in-Motion
}

\author{
Brian Mc Cormack ${ }^{1}$, Dermot Geraghty ${ }^{1}$, Margaret O’Mahony ${ }^{2}$ \\ ${ }^{1}$ Department of Mechanical and Manufacturing Engineering, \\ Trinity College Dublin, \\ Dublin 2, Ireland \\ Phone: +353 1 6083038, Fax: +353 1 6795554, E-mail: mccormb@tcd.ie \\ ${ }^{2}$ Department of Civil, Structural and Environmental Engineering, \\ Trinity College Dublin, \\ Dublin 2, Ireland
}

\begin{abstract}
This paper presents the design and manufacture of Surface Acoustic Wave (SAW) strain sensors which can be produced in a university environment. These sensors can be interrogated wirelessly and operated without a power supply, which allows for greater measurement flexibility than conventional strain gauge systems.

The design of an optimised sensor requires accurate experimental data on a number of process parameters and configurations, which for this project requires the testing of several prototype designs. The large prototyping costs typical of SAW devices can be significantly reduced if the design and manufacturing processes are integrated. This paper outlines such a system, where device function and processing issues are integrated at each stage. The result is a range of sensors which have been "designed for manufacture", optimising both the variety of devices per wafer and the yield.
\end{abstract}

Keywords - Weigh-in-Motion, SAW, sensor design

\section{INTRODUCTION}

New sensor technology is an important aspect of future Intelligent Transportation Systems (ITS), which aim to best utilise existing infrastructure and provide sustainable transport solutions. A practical Weigh-in-Motion (WIM) system (i.e. one which calculates the gross vehicle weights of heavy vehicles travelling at highway speeds [1]) could be a key ITS application, providing:

1. Vehicle taxation based on gross vehicle weight, distance travelled, exhaust emission class etc.

2. Regulatory enforcement of maximum vehicle weight, to improve public safety.

3. Fleet management for hauliers.

4. Vehicle condition monitoring for preventative maintenance.

5. Point of delivery weighing and billing of bulk loads.

To date, no low-cost WIM system has been available for real-time measurements, i.e., at arbitrary positions on the road network. Existing systems are either road-based, which only provide point measurements, or vehicle-based, which require expensive transducers and long installation times.

In this project, it is proposed that strain-sensitive Surface Acoustic Wave (SAW) sensors will be used to measure axle strain on Heavy Goods Vehicles in real-time, and that this will be used to infer the gross weight of the vehicle. SAW sensors can be operated both passively and wirelessly [2],[3], and are well suited to automotive applications [4],[5]. An added advantage is that the sensors can operate within a wide temperature range, which is essential for operation in extreme climates. Figure 1 shows the proposed system layout, as implemented in a fleet management system for hauliers. Each SAW sensor is networked using a wireless RF link to a central interrogation unit, which collects and processes the strain information to calculate vehicle weight. This data is then passed via a standard interface to a commercial off-the-shelf (COTS) vehicle tracking unit, which incorporates a GSM link for data transmission to the fleet control centre. Thus the haulier can monitor both vehicle position and weight at any time.

This paper focuses on the design and manufacturing processes used to transform SAW sensor specifications into physical devices for testing. The traditional method of producing SAW devices is described, and an integrated design and manufacturing process flow is then presented. This integrated system is more suitable for low-volume research purposes in a university environment as it allows a larger variety of prototype designs to be created, and affords more visibility of the manufacturing process to aid future design work.

\section{PROPOSED APPROACH}

The design of an optimised sensor requires accurate experimental data on a number of process parameters and device configurations, which for this project requires the testing of several prototype designs. Traditionally, a client who requires a custom-designed SAW device (e.g. for a new communications 


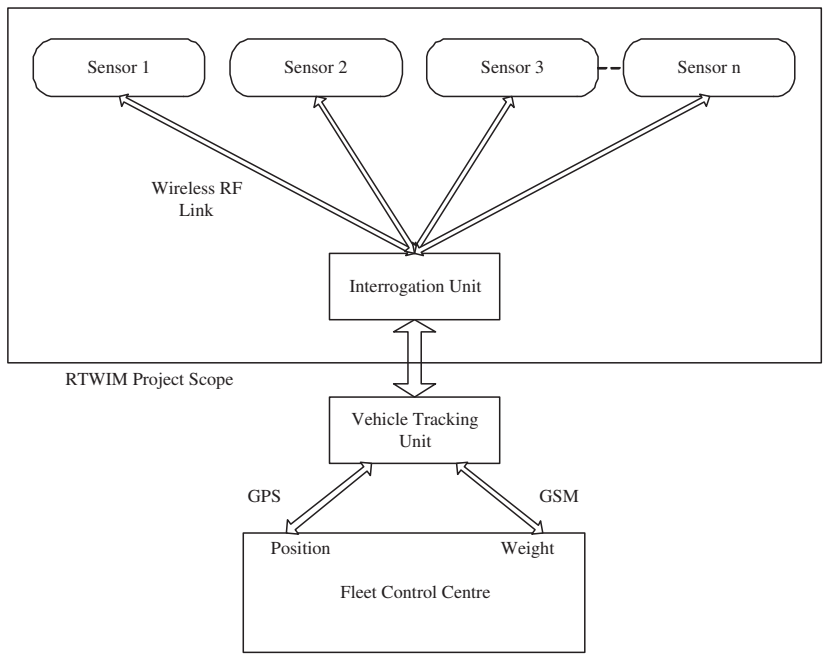

Fig. 1. Layout of Real-Time Weigh-in-Motion (RTWIM) system

application) specifies a number of important parameters (e.g. resonant frequency, amplitude of resonant peak etc.) required of the device to the SAW manufacturer, who then produces a prototype wafer of devices. These experimental devices are usually adapted from existing designs, and are tested to select the best design for production. While successful for mainstream SAW devices (which are produced in bulk and used for electronic applications), there are two major disadvantages for SAW sensor research:

1. Cost of development: the cost of a design run, which may have to be iterated as specifications change, is prohibitive for a university research project. These prototyping costs are excessive given the small production volumes used for research purposes.

2. Separation of design and manufacture: for research purposes it is essential that each stage of the project is 'visible', i.e., that the processes used can be easily examined. This is especially true of this project, as both the electrical and mechanical characteristics of the SAW sensors need to be considered. Mainstream SAW manufacturers use proprietary techniques to fine-tune device performance, effectively separating the design and manufacturing processes.

In light of these disadvantages, it was decided to implement an integrated design and manufacturing system for this project, which will allow the devices to be fabricated in a university research environment. Figure 2 shows a flowchart for the system. At each stage of the process, the different (and sometimes conflicting) requirements of design and manufacturing must be considered. This coordinated approach should reduce production problems, as the sensors have effectively been "designed for manufacture". Production costs are significantly reduced as the devices can be produced in small microelectronics laboratories, and at every stage the effect of design decisions on production can be examined.

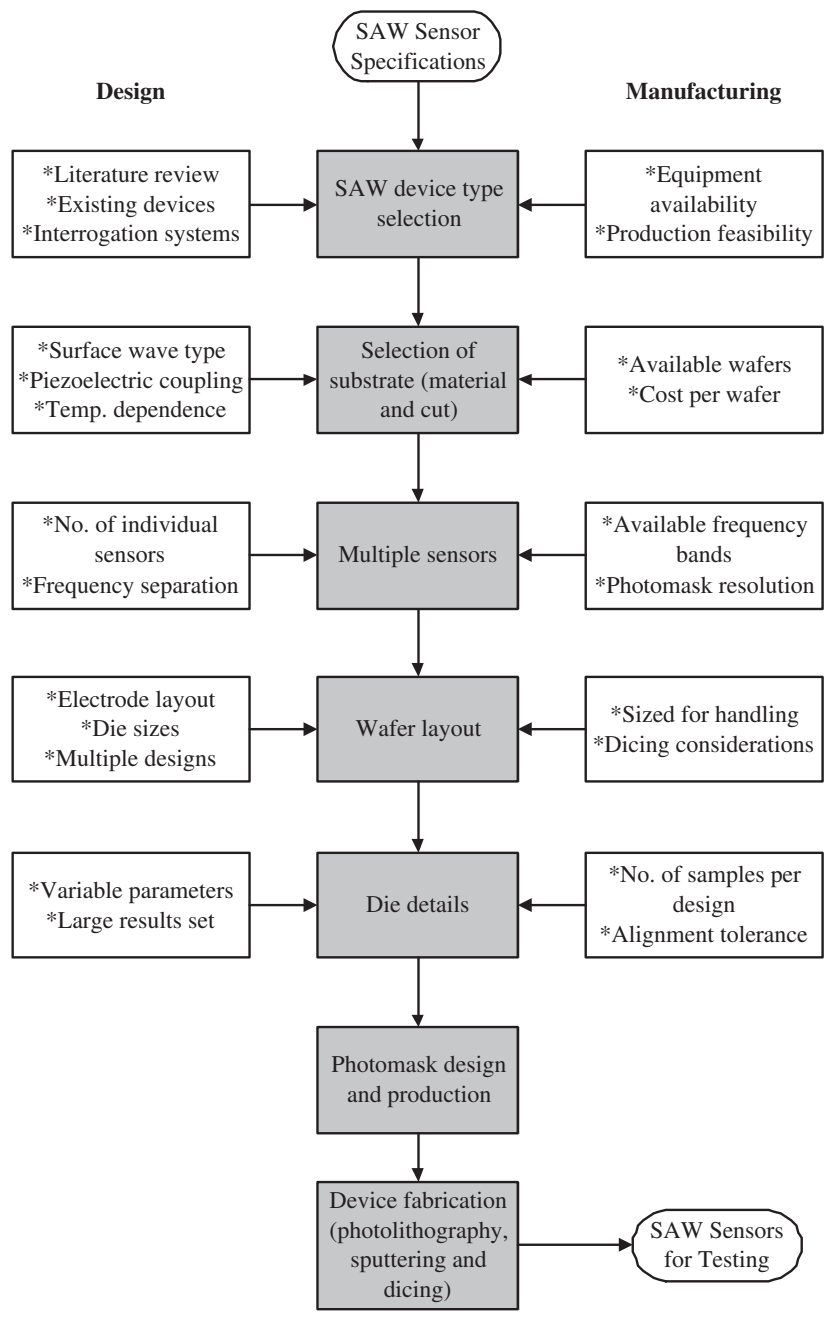

Fig. 2. Flowchart for design/manufacturing process

The initial inputs to the system are the SAW sensor specifications. These describe the sensors' behaviour both as a wireless, passive device and as a strain sensor. The wireless, passive measurement capabilities are only useful if the sensors can operate according to existing standards for low-power RF devices (e.g. [6]). Similarly, the performance of the devices as strain sensors must be comparable to that of existing foil resistive strain gauges. Following an evaluation of the specifications, the finished sensors should have the following attributes:

- Low attenuation: the sensors are passive, and thus insertion attenuation must be minimised to return the maximum signal power.

- Selective bandwidth: due to fabrication constraints and European frequency regulations [6], the devices are restricted to wireless operation within the European ISM band of $433.05-434.79 \mathrm{MHz}$, which is quite narrow for a multi-sensor environment. Strain sensitivity must be such that the measurand can be detected over the operating range, without interfering with the response from 
other sensors.

- Clearly defined sensor response: the RF channel is likely to be noisy, with interference from the vehicle and from other devices in the frequency band.

- Consistent, space-independent interrogation: the multiple sensors will be distributed around the vehicle, and thus will have different signal paths, lines-of-sight etc. The position of the sensor should not affect the resolution of the measurement.

The subsequent process steps are described below:

1. SAW device type selection: the first process stage is the choice of SAW device to be developed as a sensor. Basic device types include SAW resonators and SAW delay lines [7]. The 1-port SAW resonator was chosen for development, as it combines narrowband operation, low device attenuation, low interrogation system cost [8] and small die size: all of these characteristics are required for the RTWIM sensors. The manufacturing steps for most types of SAW device are common, and thus the performance of the available photolithography equipment (a Karl Süss MJB3 mask aligner) is the only input here.

2. Substrate selection: Rayleigh-type SAW were chosen as the wave type for the sensors, and for optimum performance the piezoelectric coupling should be maximised and temperature dependence minimised. For multiple sensor operation within the ISM band, narrowband behaviour is preferable. ST-cut quartz displays both low temperature dependence at room temperature [9] and narrowband piezoelectric performance. The piezoelectric coupling can be maximised by appropriate choice of propagation direction $\left(0^{\circ}\right.$ and $35^{\circ}$ in this case) [10]. Many sizes of wafer are available at reasonable cost.

3. Multiple sensors: several sensors will be required in the measurement system, and this must be considered early in the design cycle. As the SAW resonator was chosen for development, Frequency Division Multiple Access (FDMA) [11] may be used for interrogation of multiple devices. The operating frequency of each sensor is largely dependent on the SAW velocity $v_{s}$ (which is a function of the SAW propagation angle on the substrate) and the electrode pitch $p$ [9]. Tight control of electrode linewidth is thus needed to maximise the number of sensors in the band. In order to minimise variations in electrode widths due to rounding of dimensions, the photomask was designed to match the $8.33 \mathrm{~nm}$ address size of the photomask machine (an Etec Systems Alta3000). Three different finger widths $(1.82427,1.81594$ and $1.80761 \mu \mathrm{m})$ were chosen, which give a range of operating frequencies close to the ISM band. This choice of machine-matched dimensions will show the effect of data conversion and machine tolerance on mask dimensions: $\pm 0.05 \mu \mathrm{m}$ is expected. This spread of device frequencies will allow a wider study of the parameters affecting SAW strain sensors (see below).

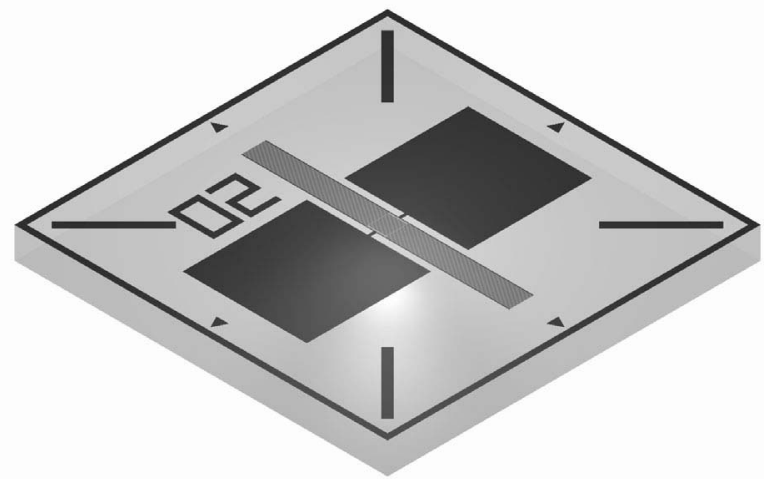

Fig. 3. Rendered view of Die 02

4. Wafer layout: modern SAW devices for communications have a surface area of c. $2 \times 1 \mathrm{~mm}$, which makes them far too small for manual handling as strain sensors. A nominal die size of $6 \times 6 \mathrm{~mm}$ was chosen for the SAW sensors, allowing compatibility with existing strain gauges, space for different electrode configurations and connection pads for external antennas and circuits. Larger die of $7.5 \times 7.5 \mathrm{~mm}$ were used for 'multiple' sensors, where several SAW resonators on a single die are used for measurement. The wafer layout is designed for easy dicing into individual sensors, and on a 3" wafer yields 48 single and 18 'multiple' sensors.

5. Die details: as the sensors have been custom designed for the project, there is scope to prototype many different varieties of device. Centre frequencies, reflector bank sizes etc. have been varied across the wafer, giving 24 single and 9 'multiple' sensor types. This will allow a novel parametric study of device specifications for SAW strain sensors.

\section{RESULTS}

The main output of this design and manufacturing process was a photomask design, and the resulting mask is being used in a lift-off photolithography process [12] to produce the SAW sensors. An example of a sample die from the photomask is shown in Figure 3. The SAW resonator structure is in the centre of the die, with connection pads on each side; orientation marks are also added for installation purposes. A section of the resonator structure is shown in Figure 4. As mentioned above, linewidth control is essential to ensure a narrow spread of device frequencies. Variations in linewidth from the original design are caused primarily by manufacturing tolerances on the photomask machine; rounding during data conversion 


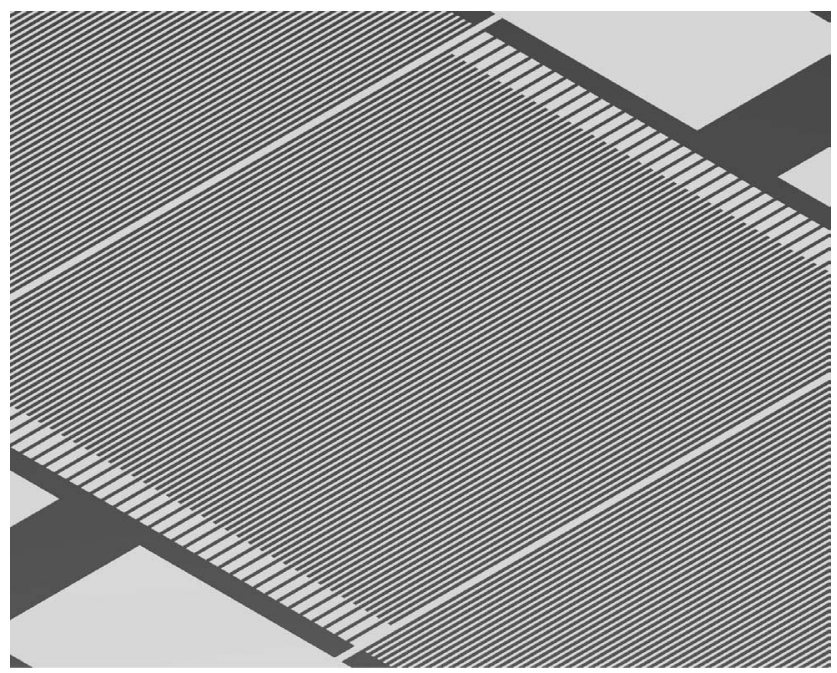

Fig. 4. Closeup of electrode structure of Die 02

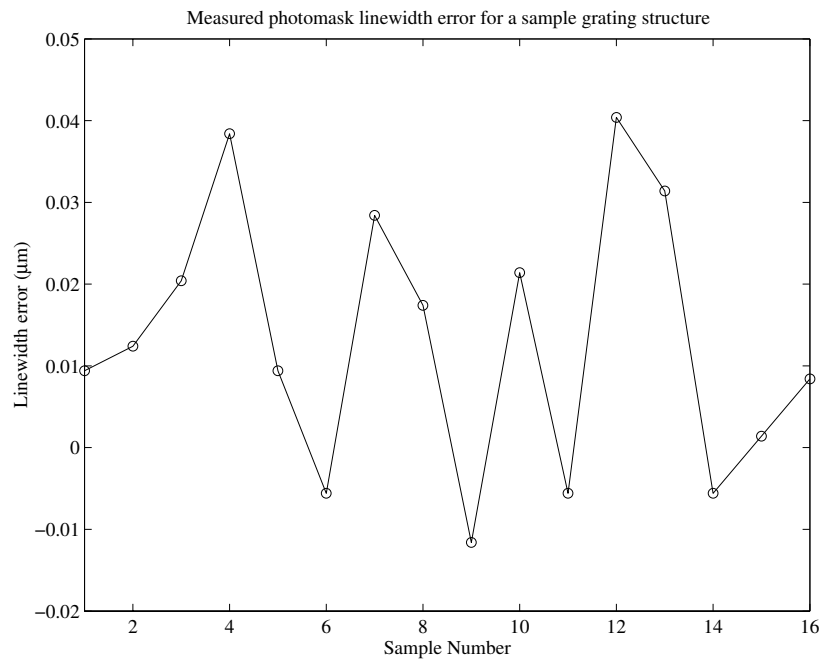

Fig. 5. Linewidth error across a grating section

accounts for a small error. Figure 5 shows the measured error in linewidth across an electrode section: nominal linewidth is $1.80761 \mu \mathrm{m}$. The photomask dimensions all lie within the specified $\pm 0.05 \mu \mathrm{m}$ tolerance. Small deviations in sensor operating frequency due to linewidth bias can be corrected by adjusting the wave propagation direction and/or the electrode thickness.

Preliminary resist patterns of a sample sensor are shown in Figure 6. Although the available manufacturing equipment was a key consideration during the design process, the exposure and development times during fabrication must still be optimised manually [13]. This optimisation is essential in order to produce the required high-definition resist patterns. The wafers can then be metallised by sputtering or evaporation to produce the sensor electrodes and grating elements. Individual die can subsequently be tested as standard SAW devices

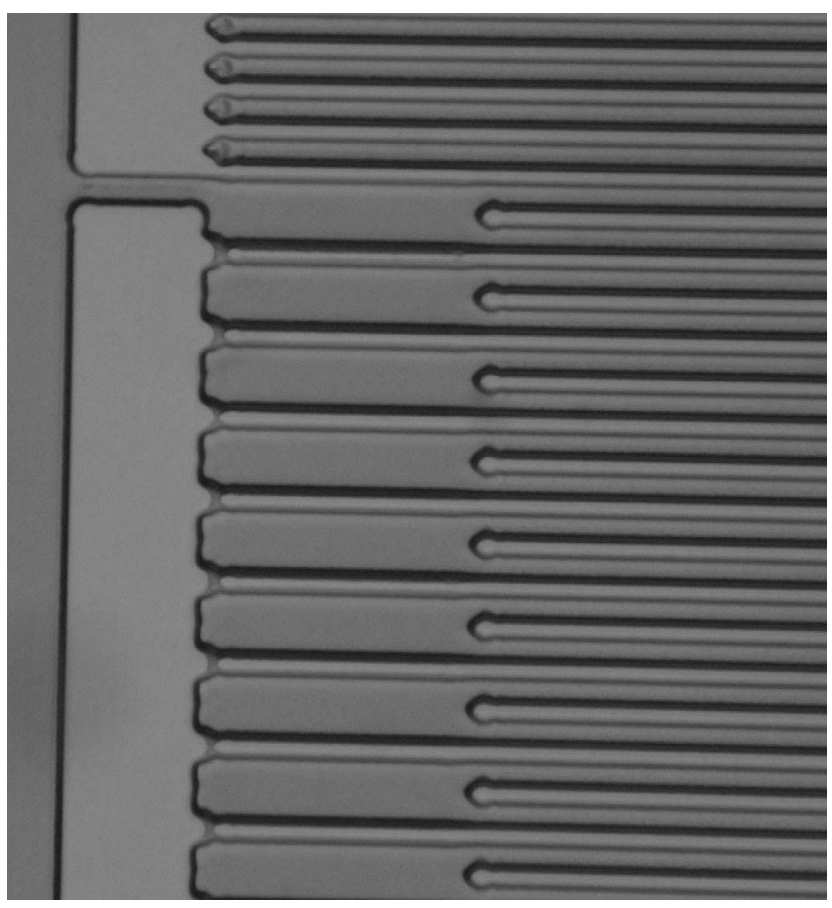

Fig. 6. Resist pattern of grating section

(e.g. for measurement of resonant frequency, quality factor, response bandwidth) before implementation as strain sensors.

Although the optimisation is still in progress, some preliminary results may be drawn. The integration of design and manufacture provides a relationship between the critical dimensions in a design and those on the actual device. This allows future designs to be designed and manufactured to tighter frequency tolerances. A consequence of this is that more sensors can be designed to operate in a narrow frequency band, which is a crucial requirement for a multi-sensor environment like RTWIM. The wide variety of device designs will provide experimental data over a range of configurations, which can then be used as parameters for device modelling techniques such as Coupling-of-Modes (COM) analysis [14],[15].

\section{CONCLUSIONS}

In summary, an integrated design and manufacturing system has been implemented for Real-Time Weigh-in-Motion SAW strain sensors. This system manages the development of SAW sensors from basic specifications to finished devices, while increasing process visibility and lowering costs relative to traditional design and manufacturing processes. The resulting devices have thus been custom designed for a demanding multi-sensor environment. 


\section{ACKNOWLEDGEMENTS}

This work is funded by the Centre for Transport Research and Innovation for People (TRIP) at Trinity College Dublin, which forms part of the HEA's PRTLI initiative. Device fabrication is carried out with the assistance of the SFI Trinity Nanoscience Laboratory.

\section{REFERENCES}

[1] D. Cebon, Handbook of Vehicle-Road Interaction. Lisse: Swets \& Zeitlinger, 1999.

[2] A. Pohl, "A review of wireless SAW sensors," IEEE Trans. Ultrason., Ferroelect., Freq. Contr., vol. 47, no. 2, pp. 317-332, 2000.

[3] J. Beckley, V. Kalinin, M. Lee, and K. Voliansky, "Non-contact torque sensors based on SAW resonators," in Proc. IEEE International Frequency Control Symposium and PDA Exhibition, 2002, pp. 202-213.

[4] B. Jacoby, H. Eisenschmid, and F. Herrmann, "The potential of microacoustic SAW- and BAW-based sensors for automotive applications - a review," IEEE Sensors J., vol. 2, no. 5, pp. 443-452, 2002.

[5] A. Pohl, R. Steindl, and L. Reindl, "The "Intelligent Tire" utilizing passive SAW sensors - measurement of tire friction," IEEE Trans. Instrum. Meas., vol. 48, no. 6, pp. 1041-1046, 1999.

[6] Radio Equipment to be used in the $25 \mathrm{MHz}$ to $1000 \mathrm{MHz}$ range with power levels ranging up to $500 \mathrm{~mW}$, European Telecommunications Standards Institute (ETSI) Std. EN 300 220, 2000.

[7] C. K. Campbell, Surface Acoustic Wave Devices for Mobile and Wireless Communications. San Diego: Academic Press, 1998.

[8] A. Pohl, G. Ostermayer, and F. Seifert, "Wireless sensing using oscillator circuits locked to remote high-Q SAW resonators," IEEE Trans. Ultrason., Ferroelect., Freq. Contr., vol. 45, no. 5, pp. 1161-1168, 1998.

[9] D. P. Morgan, Surface-Wave Devices for Signal Processing. Amsterdam: Elsevier, 1991.

[10] M. P. de Cunha and A. Y. Nakano, "Comparison between ST-cut quartz $25^{\circ}$ and $-60^{\circ}$ NSPUDT propagation directions," IEEE Trans. Ultrason., Ferroelect., Freq. Contr., vol. 49, no. 6, pp. 820-826, 2002.

[11] A. Pohl, "State of the art in signal processing for wireless SAW sensing," in Proc. Joint Meeting EFTF - IEEE IFCS, 1999, pp. 1031-1036.

[12] H. Matthews, Ed., Surface Wave Filters: Design, Construction and Use. New York: Wiley-Interscience, 1977.

[13] H. F. Thompson, C. W. Grant, and M. J. Bowden, Eds., Introduction to Microlithography, 2nd ed. Washington DC: American Chemical Society, 1994.

[14] V. Plessky and J. Koskela, "Coupling-of-modes analysis of SAW devices," Int. J. High Speed Elec. and Systems, vol. 10, no. 4, pp. 867-947, 2000.

[15] T.-T. Wu et al., "Inverse determination of coupling of modes parameters of surface acoustic wave resonators," Jpn. J. Appl. Phys., vol. 41, pp. 6610-6615, 2002. 